OPEN ACCESS

Edited by:

Marta López,

Consejo Superior de Investigaciones Cientificas (CSIC), Spain

Reviewed by:

Pierluigi Di Ciccio,

Università degli Studi di Torino, Italy

Alejandro Garrido-Maestu,

Laboratório Ibérico Internacional

de Nanotecnologia (INL), Portugal

*Correspondence:

Mei-Jun Zhu

meijun.zhu@wsu.edu

Specialty section:

This article was submitted to

Food Microbiology

a section of the journal

Frontiers in Microbiology

Received: 30 June 2018 Accepted: 07 September 2018

Published: 12 October 2018

Citation:

Korany AM, Hua Z, Green T,

Hanrahan I, El-Shinawy SH, El-kholy A, Hassan G and Zhu M-J

(2018) Efficacy of Ozonated Water,

Chlorine, Chlorine Dioxide,

Quaternary Ammonium Compounds and Peroxyacetic Acid Against Listeria

monocytogenes Biofilm on

Polystyrene Surfaces.

Front. Microbiol. 9:2296.

doi: 10.3389/fmicb.2018.02296

\section{Efficacy of Ozonated Water, Chlorine, Chlorine Dioxide, Quaternary Ammonium Compounds and Peroxyacetic Acid Against Listeria monocytogenes Biofilm on Polystyrene Surfaces}

\author{
Ahmed Mahmoud Korany 1,2, Zi Hua', Tonia Green'1, Ines Hanrahan ${ }^{3}$, \\ Saadia Helmy El-Shinawy², Adel El-kholy², Gamal Hassan² and Mei-Jun Zhu1* \\ ${ }^{1}$ School of Food Science, Washington State University, Pullman, WA, United States, ${ }^{2}$ Food Hygiene and Control \\ Department, Faculty of Veterinary Medicine, Beni-Suef University, Beni Suef, Egypt, ${ }^{3}$ Washington Tree Fruit Research \\ Commission, Wenatchee, WA, United States
}

Listeria monocytogenes contaminated processing equipment and the general packing environment have been implicated in deadly foodborne listeriosis outbreaks, highlighting the significance of proper sanitization and disinfection of food contact surfaces. This study aims to comprehensively evaluate antimicrobial efficacy of commercially available, economical sanitizers at practical concentrations against $L$. monocytogenes biofilm formed on polystyrene surfaces under different conditions. Ozonated water 1-min treatment at 1.0, 2.0, and $4.0 \mathrm{ppm}$ resulted in $\sim 0.9,3.4$, and 4.1 log reduction of L. monocytogenes single strain biofilm grown on polystyrene surfaces, respectively. However, its efficacy was dramatically diminished in multi-strain L. monocytogenes biofilm and was further compromised by aged biofilm and in the presence of organic matter. Quaternary ammonium compounds (QAC) at 100/400 ppm, chlorine at 100/200 ppm, chlorine dioxide at 2.5/5.0 ppm and peroxyacetic acid (PAA) at 80/160 ppm resulted in 2.4/3.6, 2.0/3.1, 2.4/3.8, and 3.6/4.8 log reduction of L. monocytogenes single strain biofilm, respectively. Antimicrobial efficacies of all tested sanitizers against 7-day-old biofilm were much lower when compared to 2-day-old biofilm, with PAA being the least influenced by the age of the biofilm. Organic matter conditioning with diluted milk or apple juice dramatically impacted the antimicrobial efficacy of all sanitizers. PAA treatment of $1 \mathrm{~min}$ at 160-200 ppm resulted in a 3.2-3.5 log reduction against 7-day-old biofilm in the presence of organic matter, thus showing its effectiveness in eradicating L. monocytogenes biofilm on polystyrene surface. Collectively, data highlight the importance of timely and thoroughly cleaning food contact surfaces before disinfection and provides practical information and guidance for the food industry in selecting the most effective sanitizer in their sanitizing regimes to eliminate L. monocytogenes biofilm.

Keywords: biofilm, L. monocytogenes, sanitizers, peroxyacetic acid, polystyrene, organic matter 


\section{INTRODUCTION}

Listeria monocytogenes causes nearly 1,600 illnesses in the United States annually with a $16 \%$ or higher mortality rate (Scallan et al., 2011). It is frequently incriminated in ready-to-eat meat (CDC, 1989, 1999, 2000; Currie et al., 2015), soft cheese (Heiman et al., 2016), and fresh produce (McCollum et al., 2013; Angelo et al., 2017) associated listeriosis outbreaks. It has a strong ability to form biofilms on a wide variety of abiotic surfaces commonly used in food processing facilities (Di Bonaventura et al., 2008), persisting on food processing equipment and food contact surfaces and representing a continuous contamination source (Almeida et al., 2013). Cross-contamination of foods with pathogens via contaminated equipment and contact surfaces is an important factor contributing to foodborne outbreaks (Gaulin et al., 2012; Heiman et al., 2016). The fresh produce processing equipment and environment have been implicated as important contamination sources for L. monocytogenes outbreaks (McCollum et al., 2013; Angelo et al., 2017). Thus, it is critical to effectively clean and sanitize all equipment and food-contact surfaces to eliminate L. monocytogenes in these niches.

Biofilm is composed of multiple cells attaching to the surface, which collectively produce protective extracellular polymeric substances (Buchanan et al., 2017). L. monocytogenes in biofilms displayed greater resistance to antimicrobial sanitizers than planktonic cells (Frank and Koffi, 1990; Norwood and Gilmour, 2000; Robbins et al., 2005). The susceptibility of L. monocytogenes in biofilm to disinfectants is influenced by a number of factors including strain characteristics and composition (Stopforth et al., 2002; Borucki et al., 2003; Kostaki et al., 2012; Poimenidou et al., 2016), the growth stage or age of biofilm (Stopforth et al., 2002; Saa Ibusquiza et al., 2011), the type of surfaces (Krysinski et al., 1992) as well as the cleanliness and/or the food residues/organic matter level of those surfaces (Gram et al., 2007; Saa Ibusquiza et al., 2011). Food residues/organic matter can affect bacterial biofilm formation as well as bacterial resistance to the applied sanitizers (Gram et al., 2007).

Food contact surfaces are cleaned and disinfected routinely in apple packing and similar ready-to-eat produce handling facilities. Chlorine, quaternary ammonium compounds (QAC), and peroxyacetic acid (PAA) are EPA registered and economical sanitizers commonly used for surface antimicrobial intervention in the food industry including the apple industry. Due to safety concerns about the production of carcinogenic halogenated by-products resulting from chlorinated organic compounds (Parish et al., 2003), chlorine dioxide has been used as an alternative to chlorine (Mahmoud et al., 2008). It has a higher oxidation capacity than chlorine ( $\sim 2.5$ times) (Benarde et al., 1965). Ozone is a potent antimicrobial effective at relatively low concentrations with no residual effects, and with a broadspectrum antimicrobial activity (Moore et al., 2000; Khadre et al., 2001), making it an ideal choice as a final sanitizer for food contacting surfaces.

Although chemical sanitizer intervention against L. monocytogenes biofilm on surfaces has been extensively studied, studies on their practical efficacy against biofilm under different conditions are limited. Most of sanitizers were used at a concentration much higher than practical or recommended levels (Gram et al., 2007; Minei et al., 2008; Belessi et al., 2011; Poimenidou et al., 2016). In addition, different L. monocytogenes strains, conditions, sanitizers, surfaces as well as different enumeration methodologies were used in different papers (Gram et al., 2007; Minei et al., 2008; Belessi et al., 2011; Poimenidou et al., 2016), making it impossible to provide guidance on effective sanitizers for industrial application. In the present study, using a calibrated biofilm enumeration method, we attempted to comprehensively evaluate the antimicrobial efficacy of commonly used chemical sanitizers in the food industry at practical application times and concentrations. Single- and 6-strain L. monocytogenes biofilms at different stages (age) of formation on polystyrene surfaces were tested and compared in the presence or absence of organic matter. Diluted apple juice and milk were used as organic matter to simulate persistent surface contamination on surface niches that are difficult to access and clean in a wide range of processing environments.

\section{MATERIALS AND METHODS}

\section{Listeria monocytogenes Strains and Cultures Preparation}

Listeria monocytogenes strain NRRL B-33069 (1/2a, bovine milk isolate), NRRL B-33466 (1/2b, environmental isolate), NRRL B-57618 (1/2a, human clinical isolate), NRRL B-33006 (1/2b complex, garlic isolate), NRRL B-33053 (4b, coleslaw isolate), and NRRL B-33385 (4b, human clinical isolate) were used to prepare a single strain and 6-strain cocktail inoculum. These strains were provided by the culture collection of the National Center for Agricultural Utilization Research (NRRL), USDA Agricultural Research Service (Peoria, IL, United States) and were stored at $-80^{\circ} \mathrm{C}$ in Trypticase Soy Broth [Becton, Dickinson and Company (BD), Sparks, MD, United States] with $0.6 \%(\mathrm{w} / \mathrm{v})$ yeast extract (Fisher Scientific, Pittsburgh, PA, United States) (TSBYE) and glycerol (20\%, v/v). Each culture was twice-activated in TSBYE consecutively at $35 \pm 2{ }^{\circ} \mathrm{C}$ for $24 \mathrm{~h}$ statically, then pelletized by centrifuging and suspended in Modified Welshimer's broth (MWB, HiMedia, West Chester, PA, United States) or TSB to achieve the target population density at $\sim 10^{7} \mathrm{CFU} / \mathrm{ml}$. To prepare a 6 -strain cocktail, each individual strain was activated separately, then combined at equal volumes.

\section{Biofilm Formation}

The above prepared culture of a single strain or 6-strain cocktail was transferred into each well of a sterile 96-well microtiter plate (polystyrene, Costar, Corning, NY, United States) conditioned with or without organic matter. Wells loaded with MWB or TSB broth were used as a negative control. The plates were covered with lid and incubated at $22^{\circ} \mathrm{C}$ (room temperature, RT) for 2, 4, and 7 days to grow L. monocytogenes biofilms with different ages. For each independent study, there were 6 wells per treatment. Triple independent experiments were conducted for each treatment combination. 


\section{Organic Matter Conditioning}

Organic matter/soil level of the food contact surface dramatically impacted L. monocytogenes adherence ability and its resistance to sanitizers (Papaioannou et al., 2018). To simulate harborage sites within processing plants that cannot be easily cleaned, the wells were pretreated with $100 \mu \mathrm{l}$ of 1:10 diluted apple juice or whole milk for $5 \mathrm{~min}$ at RT, then drained and dried in the biosafety cabinet for $30 \mathrm{~min}$ prior to being subjected to Listeria biofilm growth and sanitizer treatments.

\section{Biofilm Assessment by Crystal Violet Staining}

Following 2 or 4 days of incubation at RT, single strain $L$. monocytogenes (NRRL B-33069) biofilm grown in a 96-well polystyrene plate was washed with sterile water three times to remove loosely attached bacteria. The plate was air-dried at $37^{\circ} \mathrm{C}$ for $30 \mathrm{~min}$, followed by an addition of $150 \mu \mathrm{l}$ of $1 \%$ $(\mathrm{w} / \mathrm{v})$ crystal violet solution to each well and incubation at $22^{\circ} \mathrm{C}$ for $45 \mathrm{~min}$. The wells were triple washed with sterile water, air-dried for $30 \mathrm{~min}$ at $37^{\circ} \mathrm{C}$, and the crystal violet stain was dissolved by incubating with $100 \mu \mathrm{l}$ of ethyl alcohol (95\%) for $30 \mathrm{~min}$ at RT. The solubilized crystal violet was measured at $595 \mathrm{~nm}\left(\mathrm{CV}-\mathrm{OD}_{595}\right)$ using a Synergy H4 microplate Reader (BioTek ${ }^{\circledR}$ Instrument, Inc.). Each treatment had six replicates in a particular experiment, and the studies were repeated three times independently.

\section{Live/Dead Staining of Biofilm}

The biofilms formed on wells of 96-well polystyrene plate were subjected to the BacLight Live/Dead ${ }^{\circledast}$ staining kit (Molecular Probes) per the manufacture's manual. Briefly, cells in biofilms were incubated with 1:1 mixture of SYTO 9 and propidium iodide dyes in the dark for $15 \mathrm{~min}$, rinsed with phosphate buffered saline, $\mathrm{pH} 7.4$ (PBS), and then visualized using EVOS FL fluorescence microscope (Life Technologies). The excitation/emission for SYTO 9, the green-fluorescent stain, is $480 / 500 \mathrm{~nm}$ and denotes live cells with intact membranes. The excitation/emission for propidium iodide, the red-fluorescent stain, is $490 / 635 \mathrm{~nm}$ and denotes dead cells with damaged membranes.

\section{Biofilm Dislodgement and Enumeration}

Biofilm formed in a 96-well polystyrene plate was subjected to triple washing with sterile PBS. After adding $100 \mu \mathrm{l}$ of sterile PBS, biofilm in respective wells was dislodged either mechanically by scraping vigorously using a sterile tip or ultrasonically (Pitts et al., 2003). To detach biofilm ultrasonically, the 96-well plate was subjected to sonication treatment $\left(40 \mathrm{KHz}\right.$ SharperTek ${ }^{\circledR}$ ultrasonic, Pontiac, MI, United States) for $1 \mathrm{~min}$ at $22^{\circ} \mathrm{C}$, unless specified (Chavant et al., 2004), followed by pipetting and mixing thoroughly. The dislodged cells $(100 \mu \mathrm{l})$ from each well were transferred to a $1.5 \mathrm{ml}$ micro-centrifuge tube for 10 -fold serial dilutions. The appropriate dilutions were plated on TSAYE plates in duplicate and incubated at $35 \pm 2{ }^{\circ} \mathrm{C}$ for $48 \mathrm{~h}$.

\section{Biofilm Intervention Using Chemical Sanitizers}

After washing three times with $150 \mu \mathrm{l}$ of sterile PBS, biofilm cells were subjected to $100 \mu \mathrm{l}$ of the respective sanitizer treatment at selected concentration at $22^{\circ} \mathrm{C}$ for 1 min. After discarding the sanitizer, $150 \mu l$ of DeyEngley Neutralizing Broth (D/E) (Oxoid, United States) was added to neutralize any sanitizer residues. After washing two times with PBS, biofilm with or without sanitizer treatment was detached with $100 \mu \mathrm{l}$ of sterile PBS ultrasonically and enumerated as described above. For each independent study, there were six wells per treatment. Triple independent experiments were conducted for a selected treatment.

Bioside HS (EnviroTech, Modesto, CA, United States) containing 15\% PAA was used to prepare 80, 160, and 200 ppm PAA solutions. The concentration of PAA was confirmed using a titration kit (AquaPhoenix Scientific, Hanover, PA, United States). Chlorine solution (100 and $200 \mathrm{ppm}$ ) was prepared from Accu-Tab (Pace international, Wapato, WA, United States), and the $\mathrm{pH}$ was adjusted to 6.8 with $6 \mathrm{M} \mathrm{HCl}$. Quaternary ammonium compound (QAC) solutions (100-400 ppm) were prepared from STOPIT (Pace International, United States). Chlorine dioxide solutions were generated on-site using a customized chlorine dioxide generator (Pace International). The concentrations of chlorine dioxide solutions were calibrated using the chlorine dioxide Palintest kit (Palintest ${ }^{\circledR}$, Golden, CO, United States). Ozonated water was generated on-site using a Guardian Ozone generator (Guardian Manufacturing Inc., Cocoa, FL, United States). The concentration of ozonated water solution was calibrated using a titration kit (High Level Ozone Test Kit, Guardian Manufacturing Inc., Cocoa, FL, United States) and used at 1.0, 2.0 , and $4.0 \mathrm{ppm}$.

\section{Statistical Analysis}

Data were presented as mean values \pm standard error of the mean averaged from three independent experiments. Analysis of variance (ANOVA) was applied to determine significant differences between different groups at $P \leq 0.05$, using a SPSS package for Windows (Version 16).

\section{RESULTS}

\section{L. monocytogenes Biofilm Formation and Quantification}

The single strain $L$. monocytogenes biofilms were grown on polystyrene surfaces in different media for 2 or 4 days. The density of $L$. monocytogenes biofilm was markedly higher in MWB compared to TSB, while the population size of 4-dayold biofilm was similar to that of 2-day-old biofilm regardless of growth media (Figure 1A). A representative live and dead staining image of 4-day-old single strain biofilm is shown in Figure 1B, where green fluorescence indicates live cells and red fluorescence shows dead cells in the biofilm. 
A

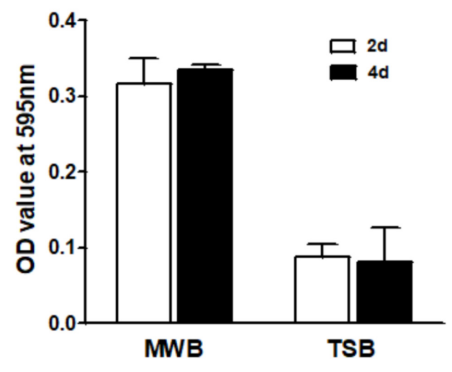

B

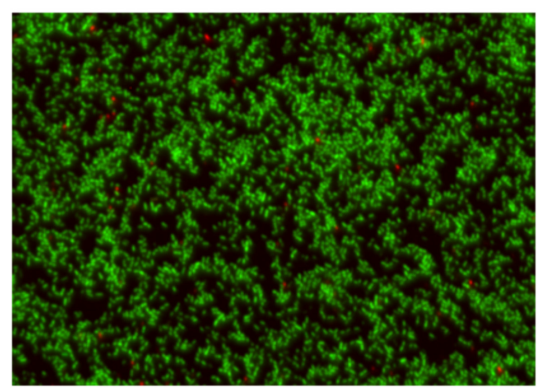

C

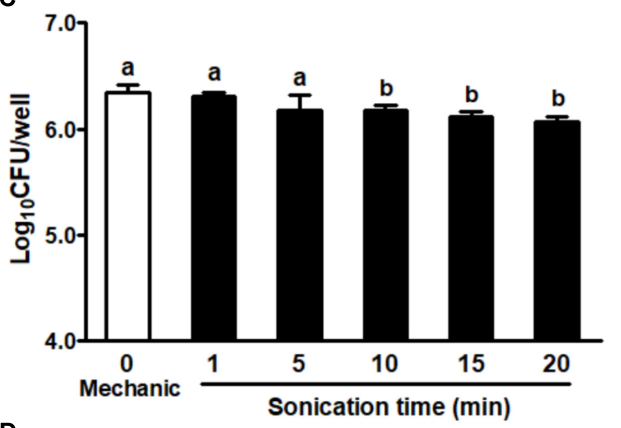

D

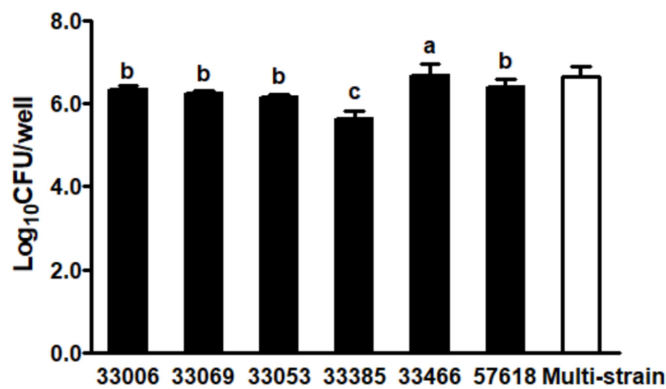

FIGURE 1 | Listeria monocytogenes single strain biofilm on polystyrene surface incubated at room temperature. (A) Quantification of single strain L. monocytogenes (NRRL B-33069) biofilm grown in MWB or TSB for 2 or 4 days by crystal violet staining; (B) BacLight Live/Dead staining of 4-day-old biofilm (NRRL B-33069) grown in MWB. Images were observed under fluorescence microscope at 100× magnification. Green, live cells; red, dead cells; (C) Enumeration of 2-day-old biofilm (NRRL B-33069) dislodged by mechanic scraping and sonication methods; (D) Enumeration of 2-day-old biofilm of different $L$. monocytogenes strains by sonication. 33006 (NRRL B-33006, 1/2b complex, garlic isolate), 33069 (NRRL B-33069, 1/2a, bovine milk isolate), 33053 (NRRL B-33053, 4 b, coleslaw isolate), 33385 (NRRL B-33385, 4b, human clinical isolate), 33466 (NRRL B-33466, 1/2b, environmental isolate), and 57618 (NRRL B-57618, 1/2a, human clinical isolate). Mean \pm SEM,

${ }^{\mathrm{a}-\mathrm{c} B a r s}$ topped with the same letter are not different at $P \leq 0.05$. Experiments were repeated independently three times.

The method for biofilm dislodgement affects data interpretation of the antimicrobial intervention efficacy. Therefore, we compared mechanical-scraping vs. 1-min sonication to dislodge cells of a 2-day-old biofilm and found that both methods had a similar detachment efficiency (Figure 1C). Sonication at $1-5 \mathrm{~min}$ resulted in similar CFU of L. monocytogenes biofilm, while sonication beyond $5 \mathrm{~min}$ decreased CFU enumeration of removed cells (Figure 1C), indicating potential cell damage during prolonged sonication. Therefore, the 1-min sonication removal method was used in the subsequent studies. We further compared the biofilm formation ability among six L. monocytogenes strains. There was no clear link between biofilm formation and the serotype of the selected strains (Figure 1D). NRRL B-33385, a 4b human clinic isolate had the lowest population density in the biofilm, while the L. monocytogenes environmental isolate (NRRL B-33466) showed the highest biofilm forming ability among all strains tested (Figure 1D).

\section{Single Strain L. monocytogenes Biofilm Inactivation by Different Sanitizers}

In general, the 2-day-old single strain L. monocytogenes biofilm was susceptible to all tested sanitizers (Figure 2). Treatment with ozonated water for $1 \mathrm{~min}$ at 1.0, 2.0, and $4.0 \mathrm{ppm}$ resulted in a $\sim 0.9,3.4$, and $4.1 \log$ reduction of single strain biofilm grown on polystyrene surfaces, respectively (Figure 2A). QAC 1min intervention at 100,200 , and $400 \mathrm{ppm}$ resulted in a $\sim 2.4$, 3.2, and $3.6 \log$ reduction of biofilm, respectively (Figure $2 \mathbf{B}$ ). Chlorine 1-min intervention at 100/200 ppm and chlorine dioxide at 2.5/5.0 ppm resulted in $\sim 2.0 / 3.1$ and $\sim 2.4 / 3.8 \mathrm{log}$ reduction of single strain $L$. monocytogenes biofilm, respectively (Figures 2C,D). PAA 1-min treatment at 80 and $160 \mathrm{ppm}$ achieved $\sim 3.6$ and $\sim 4.8 \log$ reduction of $L$. monocytogenes in single strain biofilm, respectively (Figure 2E). For each tested sanitizer, increasing concentration significantly enhanced their efficacy (Table 1).

\section{Factors Influencing Antimicrobial Efficacy of Selected Sanitizers Against Multi-Strain L. monocytogenes Biofilm}

Antimicrobial efficacy of ozonated water and QAC against a mixed strain L. monocytogenes biofilm was reduced by $1.8 \mathrm{log}$ and $0.6-0.8 \mathrm{log}$, respectively, when compared to single strain L. monocytogenes biofilm of the same age (Figure 3). However, chlorine, chlorine dioxide and PAA showed similar bactericidal effects against the 6-strain biofilm compared to single strain biofilm of the same age (Figure 3 ).

Antimicrobial efficacies of all sanitizers against 7-day-old biofilm was diminished when compared to 2-day-old biofilm (Figure 3 and Table 1), indicating mature biofilm had a higher 

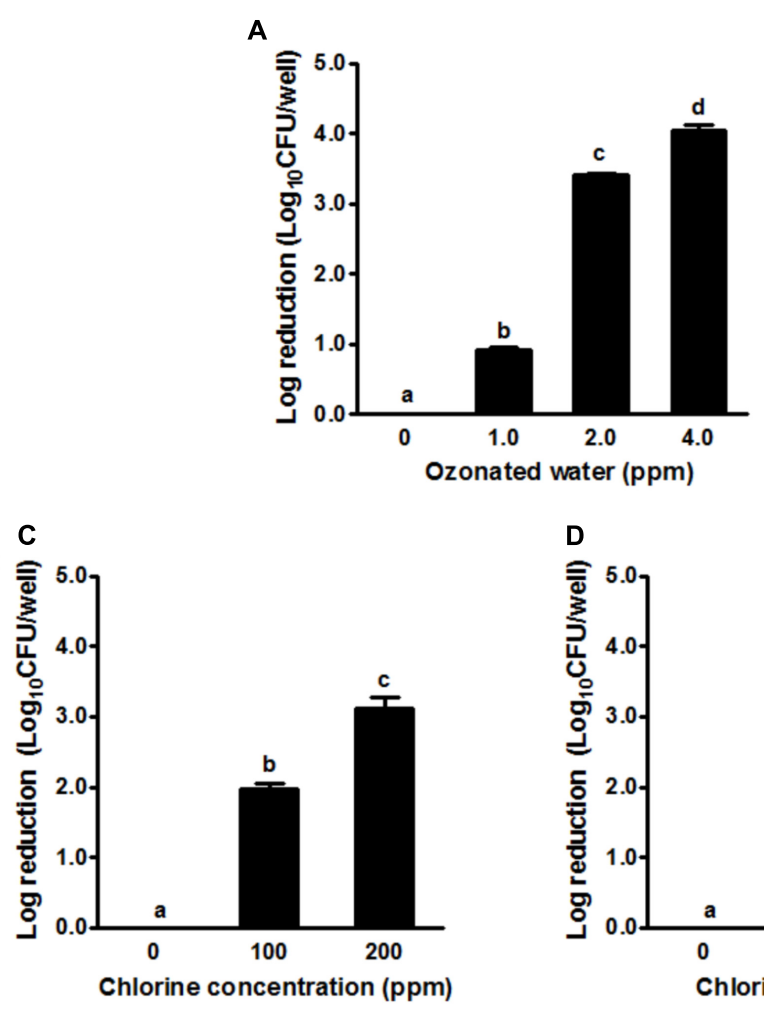

B
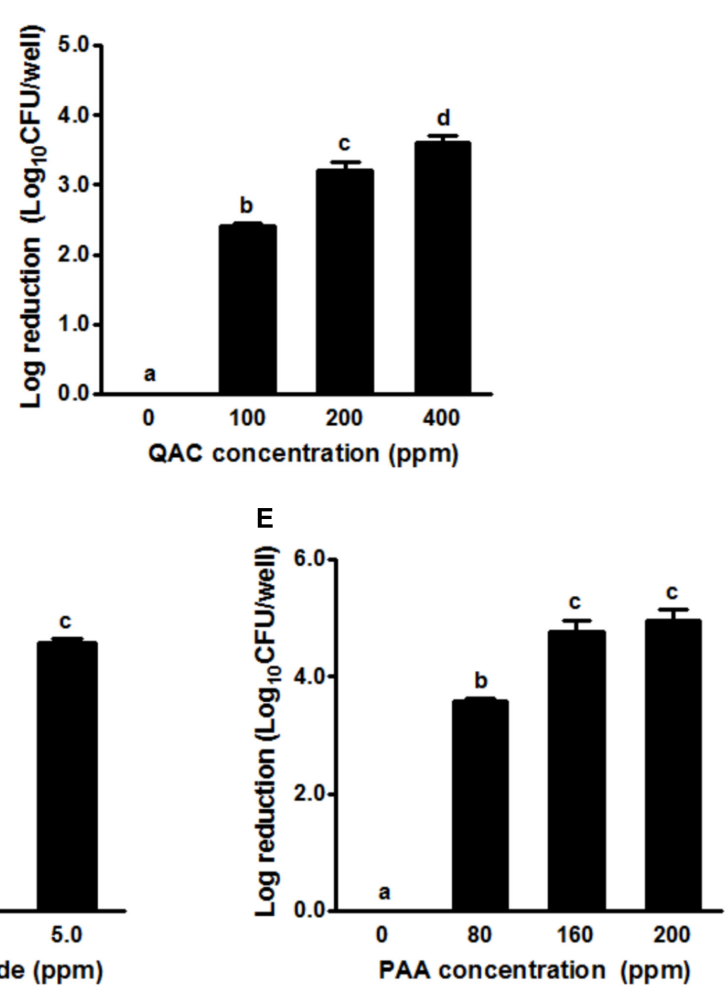

FIGURE 2 | Antimicrobial efficacy of selected sanitizer interventions against 2-day-old single strain L. monocytogenes biofilm. (A) Ozonated water; (B) Quaternary ammonium compounds (QAC); (C) Chlorine; (D) Chlorine dioxide; (E) Peroxyacetic acid (PAA). Mean \pm SEM, ${ }^{a-d}$ Bars topped with the same letter are not different at $P \leq 0.05$. Experiments were repeated independently three times.

resistance to sanitizer interventions. Antimicrobial efficacy of ozonated water and chlorine dioxide was the most influenced by the age of the biofilm (Figures 3A,D). There was about a 1.6 and $1.8 \mathrm{log}$ net reduction for $4.0 \mathrm{ppm}$ ozonated water and $5.0 \mathrm{ppm}$ chlorine dioxide, respectively, and a $1.0 \mathrm{log}$ net reduction of biocidal effects for chlorine and QAC when treating the 7-dayold biofilm (Figures 3A-D). The efficacy of PAA was relatively less impacted by the age of the biofilm (Figure 3E). There was only 0.2 and $0.5 \log$ net reduction for $80 \mathrm{ppm}$ and $160-200 \mathrm{ppm}$ PAA, respectively (Figure 3E and Table 1).

Conditioning of the surface with organic matter of apple origin further counter-acted the biocidal effects of all sanitizers against biofilm regardless of biofilm age (Figure 4 and Table 1). Ozonated water had a minimal efficacy against L. monocytogenes in both 2-day-old and 7-day-old biofilm in the presence of organic matter with apple origin (Figure 4A). Chlorine at 200 ppm, 5.0 ppm chlorine dioxide, and 400 ppm QAC were able to reduce 6 -strain $L$. monocytogenes biofilm by $1.6,1.3$, $1.6 \mathrm{log}$, respectively, for 7 -day-old biofilm, and by $2.5,2.2$, and $2.2 \mathrm{log}$, respectively, for 2-day-old biofilm (Figures 4BD). PAA 1-min treatment at 160-200 ppm resulted in 3.4$3.5 \log$ reduction against 7 -day-old biofilm conditioned with organic matter from apple origin (Figure 4E and Table 1). Similarly, surface conditioning with organic matter from milk origin impaired antimicrobial intervention against both 2-dayold and 7-day-old biofilm. Soiling from milk caused slightly more net reduction in biocidal efficacy in general compared to organic matter from apple origin (Figure 5 and Table $\mathbf{1}$ ).

\section{DISCUSSION}

\section{Strain Variability in Biofilm Formation and Quantification}

Listeria monocytogenes, as a true environmental species, is prevalent in many produce-associated locales and operations. Food processing equipment and environment have been identified as important contamination sources for cantaloupe and caramel apple listeriosis outbreaks (McCollum et al., 2013; Angelo et al., 2017). This highlights the importance of proper and effective sanitization of food contact surfaces.

Different L. monocytogenes strains have distinctive abilities to form biofilm (Borucki et al., 2003). To compensate the strain variability of biofilm formation and antimicrobial resistance to individual sanitizer a 6-strain L. monocytogenes biofilm containing three serotypes that have caused the majority of human listeriosis $(1 / 2 \mathrm{a}, 1 / 2 \mathrm{~b}$, and $4 \mathrm{~b}$ ) (Silk et al., 2012) from different food and environmental sources were used in this study. As reported in a previous study (Borucki et al., 2003), there was a strain variability in biofilm formation. However, there was no clear relationship between biofilm and serotype, which is consistent with a previous report (Borucki et al., 2003). 

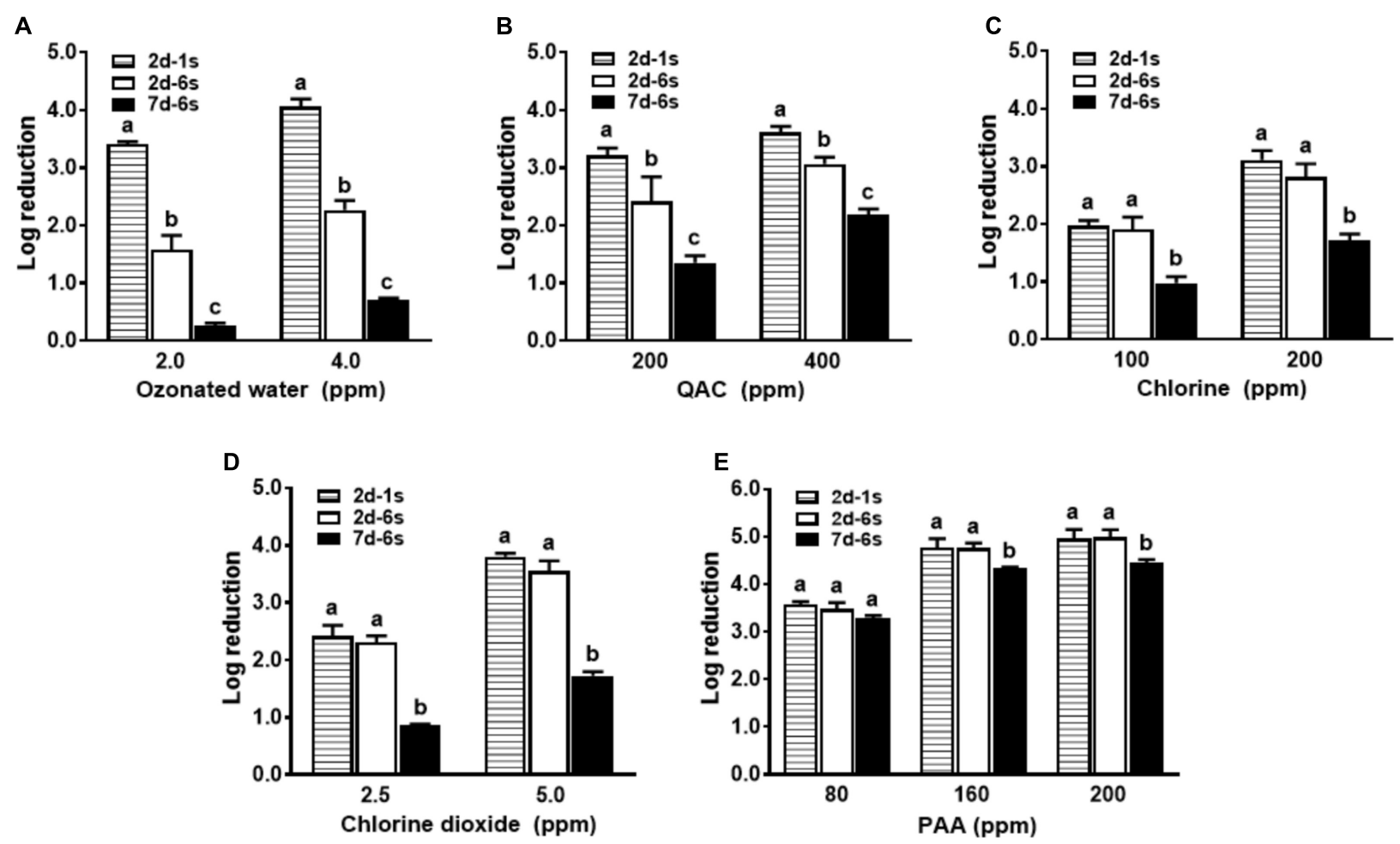

FIGURE 3 | Antimicrobial efficacy of selected sanitizer interventions against L. monocytogenes biofilms at different ages. 2d-1s, 2-day-old single strain biofilm; 2d-6s, 2-day-old 6-strain biofilm; 7d-6s, 7-day-old 6-strain biofilm. (A) Ozonated water; (B) Quaternary ammonium compounds (QAC); (C) Chlorine; (D) Chlorine dioxide; (E) Peroxyacetic acid (PAA). Mean \pm SEM, ${ }^{a-c}$ Bars topped with the same letter at the same concentration are not different at $P \leq 0.05$. Experiments were repeated independently three times.

TABLE 1 | Log reduction of 6-strain biofilm on polystyrene surface conditioned with or without organic matter post sanitizer treatments.

\begin{tabular}{|c|c|c|c|c|c|c|c|}
\hline Sanitizers & Conc. & $2 d$ & 2d-apple & 2d-milk & $7 d$ & 7d-apple & 7d-milk \\
\hline \multirow[t]{2}{*}{ Ozonated water } & 2.0 ppm & $1.58 \pm 0.14^{\mathrm{Aa}}$ & $0.12 \pm 0.03^{\mathrm{Ba}}$ & $0.20 \pm 0.06^{\mathrm{Ba}}$ & $0.26 \pm 0.05^{\mathrm{Ba}}$ & $0.11 \pm 0.04^{\mathrm{Ba}}$ & $0.16 \pm 0.02^{\mathrm{Ba}}$ \\
\hline & 4.0 ppm & $2.27 \pm 0.09^{A b c}$ & $0.36 \pm 0.05^{\mathrm{Ba}}$ & $0.31 \pm 0.05^{\mathrm{Ba}}$ & $0.70 \pm 0.04^{\mathrm{Cb}}$ & $0.24 \pm 0.05^{\mathrm{Bab}}$ & $0.29 \pm 0.03^{\mathrm{Ba}}$ \\
\hline \multirow[t]{2}{*}{ Chlorine } & 100 ppm & $1.91 \pm 0.12^{\mathrm{Aab}}$ & $1.24 \pm 0.05^{\mathrm{BCbc}}$ & $1.33 \pm 0.09^{\mathrm{Bb}}$ & $0.98 \pm 0.11^{\mathrm{BCDbc}}$ & $0.86 \pm 0.10^{\mathrm{CDc}}$ & $0.71 \pm 0.03^{\mathrm{Db}}$ \\
\hline & 200 ppm & $2.81 \pm 0.14^{\mathrm{Acd}}$ & $2.51 \pm 0.10^{\mathrm{Adf}}$ & $2.57 \pm 0.11^{A c}$ & $1.72 \pm 0.11^{\mathrm{Bd}}$ & $1.61 \pm 0.12^{\mathrm{Bd}}$ & $1.42 \pm 0.13^{\mathrm{Bcd}}$ \\
\hline \multirow[t]{2}{*}{ Chlorine dioxide } & 2.5 ppm & $2.31 \pm 0.06^{\mathrm{Abc}}$ & $0.90 \pm 0.09^{\mathrm{Bb}}$ & $0.63 \pm 0.04^{\mathrm{CDad}}$ & $0.87 \pm 0.02^{\mathrm{BCb}}$ & $0.52 \pm 0.01^{\mathrm{Db}}$ & $0.50 \pm 0.05^{\mathrm{Dab}}$ \\
\hline & 5.0 ppm & $3.55 \pm 0.10^{\mathrm{Ae}}$ & $2.23 \pm 0.06^{\text {Bdef }}$ & $1.48 \pm 0.07^{\mathrm{CDb}}$ & $1.73 \pm 0.07^{\mathrm{Cd}}$ & $1.31 \pm 0.07^{\mathrm{Dd}}$ & $1.24 \pm 0.08^{\mathrm{Dc}}$ \\
\hline \multirow[t]{2}{*}{ QAC } & 200 ppm & $2.41 \pm 0.25^{\mathrm{Abc}}$ & $1.87 \pm 0.08^{\mathrm{ABce}}$ & $1.16 \pm 0.24^{\mathrm{BCbd}}$ & $1.35 \pm 0.13^{\mathrm{BCcd}}$ & $0.86 \pm 0.05^{\mathrm{Cc}}$ & $0.75 \pm 0.08^{\mathrm{Cb}}$ \\
\hline & 400 ppm & $3.06 \pm 0.07^{\mathrm{Ade}}$ & $2.20 \pm 0.08^{\text {Bdef }}$ & $2.09 \pm 0.05^{\mathrm{Bc}}$ & $2.18 \pm 0.10^{\mathrm{Be}}$ & $1.56 \pm 0.05^{\mathrm{Cd}}$ & $1.44 \pm 0.07^{\mathrm{Ccd}}$ \\
\hline \multirow[t]{3}{*}{ PAA } & 80 ppm & $3.48 \pm 0.08^{\mathrm{Ae}}$ & $2.69 \pm 0.19^{\mathrm{Bf}}$ & $2.15 \pm 0.11^{\mathrm{Cc}}$ & $3.29 \pm 0.05^{\mathrm{Af}}$ & $2.13 \pm 0.05^{\mathrm{Ce}}$ & $1.66 \pm 0.10^{\mathrm{Dd}}$ \\
\hline & 160 ppm & $4.76 \pm 0.06^{\mathrm{Af}}$ & $3.62 \pm 0.15^{\mathrm{BDg}}$ & $3.70 \pm 0.09^{\mathrm{Be}}$ & $4.34 \pm 0.02^{\mathrm{Cg}}$ & $3.36 \pm 0.08^{\mathrm{BDf}}$ & $3.17 \pm 0.08^{\mathrm{CDe}}$ \\
\hline & 200 ppm & $4.99 \pm 0.09 \mathrm{Af}$ & $3.85 \pm 0.16^{\mathrm{BDg}}$ & $3.91 \pm 0.18^{\mathrm{Be}}$ & $4.46 \pm 0.06^{\mathrm{Cg}}$ & $3.50 \pm 0.05^{\mathrm{BDf}}$ & $3.31 \pm 0.09^{\mathrm{CDe}}$ \\
\hline
\end{tabular}

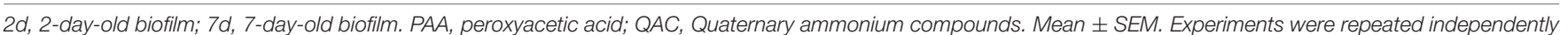

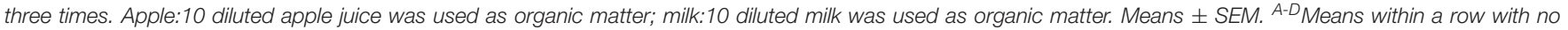
common letter differ significantly $(P \leq 0.05)$. ${ }^{a-f}$ Means within a column with no common letter differ significantly $(P \leq 0.05)$.

\section{Antimicrobial Efficacy of Ozonated Water Against $L$. monocytogenes Biofilm Formed Under Different Conditions}

Since its approval by FDA to be directly applied to food, in storage and during processing (Rice and Graham, 2001), ozone in both the aqueous and gaseous phase has been increasingly used in the food industry (Moore et al., 2000; Baumann et al.,
2009; Sheng et al., 2018). Ozone, known for its high oxidation capacity and potent antimicrobial activity, reacts with organic material 3000 times faster than chlorine (EPRI, 1997), and has the ability to attack microbial cell membranes, causing cell lysis and death. Ozonated water showed a strong efficacy against a 2-dayold single strain L. monocytogenes biofilm. A 1-min of $4.0 \mathrm{ppm}$ treatment resulted in a $4.1 \mathrm{log}$ reduction of biofilm, which was similar or higher than $200 \mathrm{ppm}$ chlorine, $400 \mathrm{ppm}$ QAC, and 


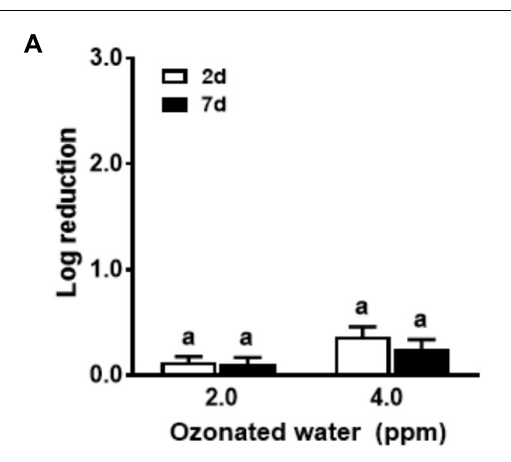

C

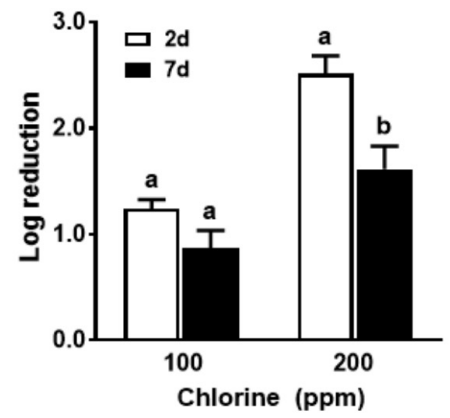

D

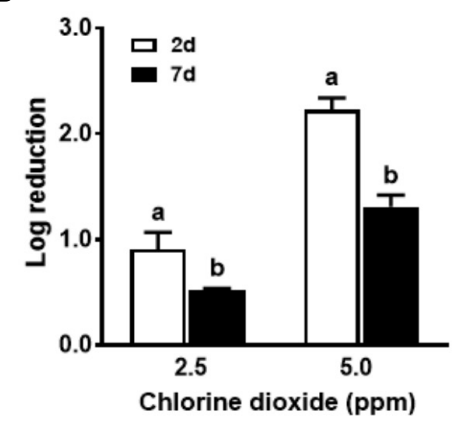

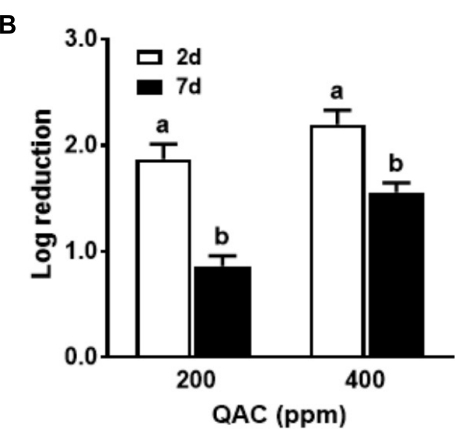

E

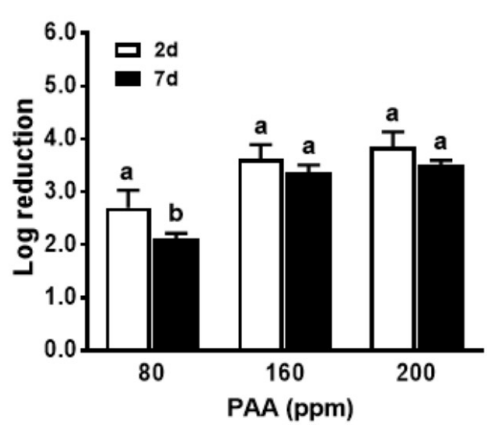

FIGURE 4 | Antimicrobial efficacy of selected sanitizer interventions against 6-strain L. monocytogenes biofilm on polystyrene surfaces in the presence of organic matter from apple. 2d, 2-day-old biofilm; 7d, 7-day-old biofilm. (A) Ozonated water; (B) Quaternary ammonium compounds (QAC); (C) Chlorine; (D) Chlorine dioxide; (E) Peroxyacetic acid (PAA). Mean \pm SEM, ${ }^{\text {a,b }}$ Bars topped with the same letter at the same concentration are not different at $P \leq 0.05$. Experiments were repeated independently three times. 1:10 diluted apple juice was used as organic matter.

5.0 ppm chlorine dioxide at the same exposure time. Similarly, 1-min $1.0 \mathrm{ppm}$ ozonated water exposure resulted in $4.2 \mathrm{log}$ reduction in a 4-day-old single strain $L$. monocytogenes biofilm on stainless steel (Baumann et al., 2009). However, the bactericidal effect of ozonated water against multi-strain L. monocytogenes biofilm was dramatically reduced and further compromised by increased age and soiling by organic matter from apple or milk origin. In support of our findings, the presence of organic matter from either milk or meat broth decreased the net log reduction of gaseous ozone (4.0 ppm for $4 \mathrm{~h}$ ) against Listeria innocua, a non-pathogenic surrogate of L. monocytogenes, biofilm on the stainless steel surface from $\sim 3.0 \mathrm{log}$ reduction to less than 1.0 or even $0.5 \mathrm{log}$ reduction, respectively (Moore et al., 2000). The reduced disinfection rate of ozonated water against the 6-strain and/or the aged biofilm was likely due to the complexity of biofilm structure or exopolysaccharide layer of the aged and/or multi-strain biofilm, and thus poor penetration or reaction with ozone. It might also be due to differential antimicrobial sensitivities of different stages of L. monocytogenes in biofilm (Chavant et al., 2004). The counter-acting/suppressive effects of organic matter on ozone efficiency is likely due to their high reactivity with ozone thus competing with bacteria. It might also result from physical shielding effects of food residues. However, the organic matter soiling had a less impact on the efficacy of ozone against Staphylococcus aureus biofilm on stainless steel surface (Moore et al., 2000). Collectively, although ozone is a potent antimicrobial, its bactericidal activity is compromised against the aged 6-strain biofilm in the presence of organic matter, highlighting practical challenges and the importance of intelligent application of ozonated water for surface disinfection. It is highly desirable for ozonated water disinfection to be preceded by more frequent and effective cleaning to remove food residue and organic matter to maximize its efficacy.

\section{Antimicrobial Efficacy of Chlorine, Chlorine Dioxide, QAC, and PAA Against L. monocytogenes Biofilm Formed Under Different Conditions}

Similar to the ozonated water, QAC was less effective against the 6-strain biofilm compared to single strain biofilm, but multistrain had a minor impact on antimicrobial efficacy of chlorine, chlorine dioxide and PAA. The antimicrobial efficacy of sanitizers against mature biofilm was decreased by $0.6-1.8$ log depending on sanitizers and their concentrations. Besides ozonated water, chlorine dioxide efficacy was most significantly impacted by the age of biofilm. In support, 200 ppm QAC/chlorine, or 150 ppm PAA at a 2-min contact were found to be less bactericidal against the bacteria in 7-day-old or 14-day-old biofilm of total microbial or mixed-strain (including L. monocytogenes and background microflora), on stainless steel surface conditioned with beef wash rinse than 2-day-old biofilm (Stopforth et al., 2002). Increased resistance against nisin, a bacteriocin, and PAA was seen in 11-day-old biofilm compared to a 4-day-old L. monocytogenes 

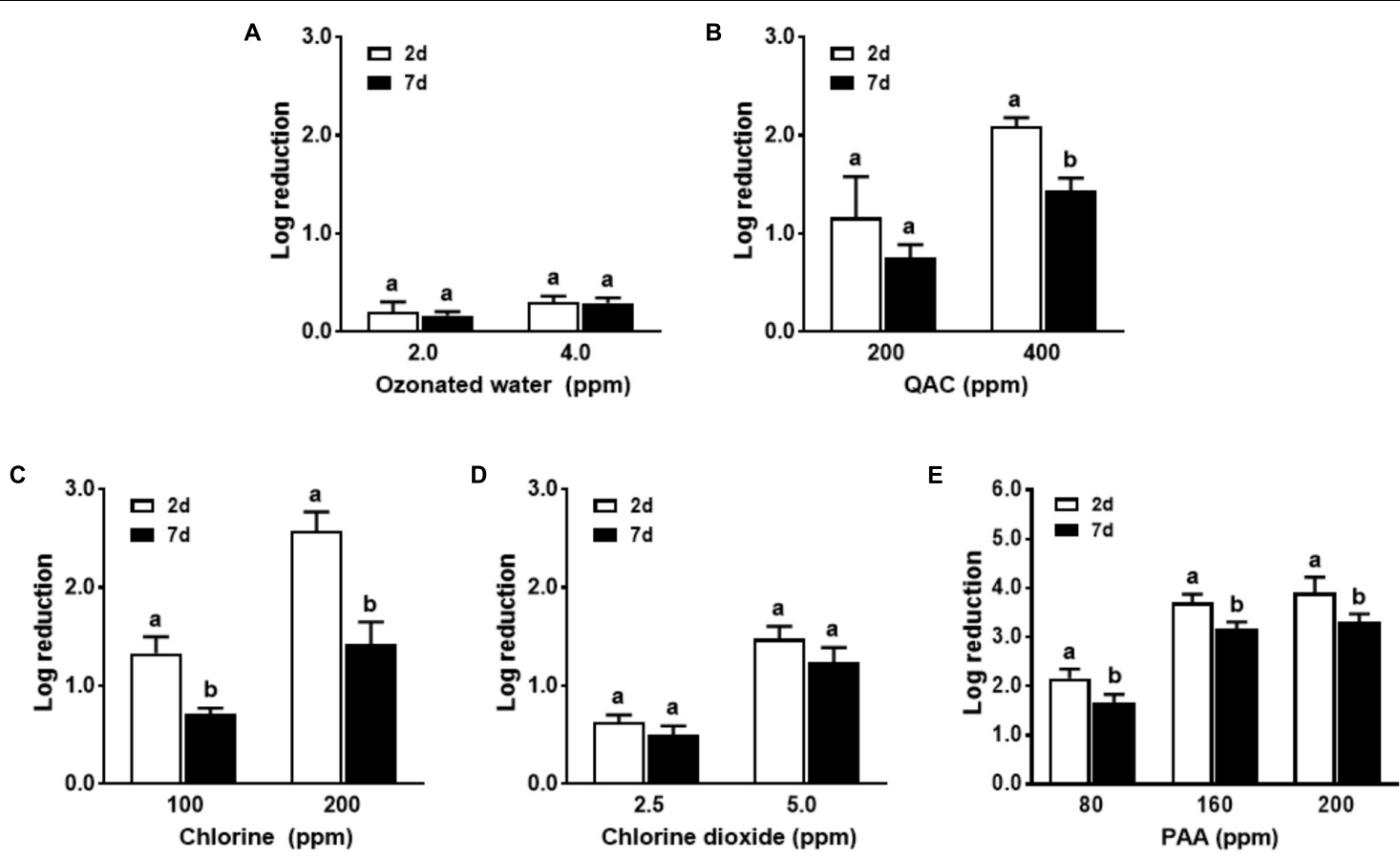

FIGURE 5 | Antimicrobial efficacy of selected sanitizer interventions against 6-strain L. monocytogenes biofilm on polystyrene surfaces in the presence of organic matter from milk. 2d, 2-day-old biofilm; 7d, 7-day-old biofilm. (A) Ozonated water; (B) Quaternary ammonium compounds (QAC); (C) Chlorine; (D) Chlorine dioxide; (E) Peroxyacetic acid (PAA). Mean \pm SEM, ${ }^{a, b}$ Bars topped with the same letter at the same concentration are not different at $P \leq 0.05$. Experiments were repeated independently three times. 1:10 diluted whole milk was used as organic matter.

biofilm (Saa Ibusquiza et al., 2011). Similarly, resistance of L. monocytogenes in biofilm on stainless steel surface against 4640 ppm chlorine, 20000 ppm PAA, and 10000 ppm QAC was considerably higher with increased age of biofilm (Belessi et al., 2011).

Soiling of polystyrene surfaces with diluted milk or apple juice further reduced bactericidal effects of all sanitizers. In combination, PAA is the most effective sanitizer treatment against the aged biofilm on the soiled polystyrene surface, which achieved a 3.2-3.5 log reduction after 1-min exposure to 160-200 ppm PAA. In agreement with our data, PAA at a range of 80 to $320 \mathrm{ppm}$ was shown to be more effective than the same concentration of chlorine in inactivation of L. monocytogenes in a 2-day-old L. monocytogenes and Pseudomonas spp. multi-species biofilm in the presence of milk origin organic matter (Fatemi and Frank, 1999). Organic matter conditioning, especially from meat emulsion, greatly counteracted biocidal effects of PAA, chlorine and QAC against L. monocytogenes biofilm on stainless steel surface (Gram et al., 2007).

The antimicrobial efficacy of PAA and QAC detected in this study was higher or comparable to the previous studies, where 5-min exposure to $2000 \mathrm{ppm}$ PAA and $500 \mathrm{ppm}$ QAC led to a 1.5-3.6 and 1.4-3.7 $\mathrm{Log} \mathrm{CFU} / \mathrm{cm}^{2}$ reduction of 3-day-old L. monocytogenes biofilm on polystyrene surface, respectively, depending on L. monocytogenes strain (Poimenidou et al., 2016). A 1-min and 10-min exposure to $7.0 \mathrm{ppm}$ chlorine dioxide resulted in a 2.3 and $3.7 \mathrm{Log} \mathrm{CFU} / \mathrm{cm}^{2}$ reduction of 4-day-old 5-strain L. monocytogenes biofilm on stainless steel coupons, respectively (Vaid et al., 2010), in contrast to 3.6 and 1.7 Log CFU/well reduction of 2-day-old and 7-day-old biofilm on polyester surface, respectively, caused by 1-min exposure to 5.0 ppm chlorine dioxide.

Data collaboratively indicated that PAA tended to be a viable sanitizer for the polystyrene surface disinfection in the food industry. The bactericidal effects of PAA might be due to its high reactivity and oxidizing power in combination with its low molecular size and high decomposition rate, which facilitates its penetration into biofilm matrix (Saa Ibusquiza et al., 2011). Biocidal effects of PAA against biofilm of L. monocytogenes and other foodborne pathogens on different processing and foodcontact surfaces warrant further research. It was reported that L. monocytogenes biofilm on stainless steel surface had a lower population density and a higher susceptibility to PAA and QAC disinfection compared to polystyrene surface (Poimenidou et al., 2016).

\section{CONCLUSION AND PERSPECTIVES}

In general, all sanitizers tested in this study at the concentrations commonly used in the food industry showed a strong bactericidal effect against 2-day-old single strain L. monocytogenes biofilm. PAA at 160-200 ppm showed a larger net log reduction compared to other sanitizers tested. The antimicrobial efficacy of all sanitizers was impaired when the biofilm was aged, and/or a 
soiled surface was present. This diminished effect was the most significant for ozonated water, followed by chlorine dioxide, QAC and chlorine, while PAA was the least influenced. A 1min treatment of 160-200 ppm PAA resulted in 3.2-3.5 log reduction of the aged biofilm on polystyrene surface with organic matter.

Our data illustrate the importance of establishing and maintaining a good cleaning process prior to sanitizer disinfection in the food industry. Food residues or soiling by organic matter, regardless of sources, diminished efficiency of sanitizers against pathogenic bacteria in general, although some sanitizers are less impacted. It is highly desirable to clean and remove organic matter/soiling effectively prior to sanitizer disinfection to maximize their efficacy. Results from this study also reflected the significance of periodical application of sanitizers to avoid establishment of the aged biofilm, which was much more difficult to be eradicated compared to the fresh one. This study provides critical and practical information for the food industry in selecting sanitizers in their surface disinfectant regimes for delicate processing niches.

\section{REFERENCES}

Almeida, G., Magalhaes, R., Carneiro, L., Santos, I., Silva, J., Ferreira, V., et al. (2013). Foci of contamination of Listeria monocytogenes in different cheese processing plants. Int. J. Food Microbiol. 167, 303-309. doi: 10.1016/j. ijfoodmicro.2013.09.006

Angelo, K. M., Conrad, A. R., Saupe, A., Dragoo, H., West, N., Sorenson, A., et al. (2017). Multistate outbreak of Listeria monocytogenes infections linked to whole apples used in commercially produced, prepackaged caramel apples: United States, 2014-2015. Epidemiol. Infect. 145, 848-856. doi: 10.1017/ S0950268816003083

Baumann, A. R., Martin, S. E., and Feng, H. (2009). Removal of Listeria monocytogenes biofilms from stainless steel by use of ultrasound and ozone. J. Food Prot. 72, 1306-1309. doi: 10.4315/0362-028X-72.6.1306

Belessi, C. E., Gounadaki, A. S., Psomas, A. N., and Skandamis, P. N. (2011). Efficiency of different sanitation methods on Listeria monocytogenes biofilms formed under various environmental conditions. Int. J. Food Microbiol. 145(Suppl. 1), S46-S52. doi: 10.1016/j.ijfoodmicro.2010.10.020

Benarde, M. A., Israel, B. M., Oliveri, V. P., and Granstrom, M. L. (1965). Efficiency of chlorine dioxide as a bactericide. Appl. Microbiol. 13, 776-780.

Borucki, M. K., Peppin, J. D., White, D., Loge, F., and Call, D. R. (2003). Variation in biofilm formation among strains of Listeria monocytogenes. Appl. Environ. Microbiol. 69, 7336-7342. doi: 10.1128/AEM.69.12.7336-7342.2003

Buchanan, R. L., Gorris, L. G. M., Hayman, M. M., Jackson, T. C., and Whiting, R. C. (2017). A review of Listeria monocytogenes: an update on outbreaks, virulence, dose-response, ecology, and risk assessments. Food Control 75, 1-13. doi: 10.1016/j.foodcont.2016.12.016

CDC (1989). Listeriosis associated with consumption of turkey franks. MMWR Morb. Mortal. Wkly. Rep. 38, 267-268.

CDC (1999). Update: multistate outbreak of listeriosis-United States, 1998-1999. MMWR Morb. Mortal. Wkly. Rep. 47, 1117-1118.

CDC (2000). Multistate outbreak of listeriosis-United States, 2000. MMWR Morb. Mortal. Wkly. Rep. 49, 1129-1130.

Chavant, P., Gaillard-Martinie, B., and Hebraud, M. (2004). Antimicrobial effects of sanitizers against planktonic and sessile Listeria monocytogenes cells according to the growth phase. FEMS Microbiol. Lett. 236, 241-248. doi: 10.1111/j.1574-6968.2004.tb09653.x

Currie, A., Farber, J. M., Nadon, C., Sharma, D., Whitfield, Y., Gaulin, C., et al. (2015). Multi-province Listeriosis outbreak linked to contaminated deli meat consumed primarily in institutional settings. Canada, 2008. Foodborne Pathog. Dis. 12, 645-652. doi: 10.1089/fpd.2015.1939

\section{AUTHOR CONTRIBUTIONS}

$\mathrm{AK}$ and $\mathrm{M}-\mathrm{JZ}$ designed the experiments. $\mathrm{AK}, \mathrm{ZH}$, and TG performed the experiments. M-JZ and AK wrote and revised the manuscript. IH, SE-S, AE-k, and GH edited the manuscript.

\section{FUNDING}

This study was supported by Washington Tree Fruit Research Commission. AK was supported by Egyptian Government Research Scholarship, Egypt, Cultural and Educational Bureau, the Arab Republic of Egypt.

\section{ACKNOWLEDGMENTS}

We thank the Guardian Ozone for their generous donation of Ozone Generator, and the Pace International Inc., for providing Accu-Tab, Bioside HS, STOP-IT as well as the Chlorine Dioxide Generator.

Di Bonaventura, G., Piccolomini, R., Paludi, D., D’Orio, V., Vergara, A., Conter, M., et al. (2008). Influence of temperature on biofilm formation by Listeria monocytogenes on various food-contact surfaces: relationship with motility and cell surface hydrophobicity. J. Appl. Microbiol. 104, 1552-1561. doi: 10.1111/j. 1365-2672.2007.03688.x

EPRI (1997). Ozone-GRAS affirmation for use in food. Food Ind. Curr. 1, 1-6.

Fatemi, P., and Frank, J. F. (1999). Inactivation of Listeria monocytogenes/Pseudomonas biofilms by peracid sanitizers. J. Food Prot. 62, 761-765. doi: 10.4315/0362-028X-62.7.761

Frank, J. F., and Koffi, R. A. (1990). Surface-adherent growth of Listeria monocytogenes is associated with increased resistance to surfactant sanitizers and heat. J. Food Prot. 53, 550-554. doi: 10.4315/0362-028X-53.7.550

Gaulin, C., Ramsay, D., and Bekal, S. (2012). Widespread listeriosis outbreak attributable to pasteurized cheese, which led to extensive cross-contamination affecting cheese retailers. Quebec, Canada, 2008. J. Food Prot. 75, 71-78. doi: 10.4315/0362-028X.JFP-11-236

Gram, L., Bagge-Ravn, D., Ng, Y. Y., Gymoese, P., and Vogel, B. F. (2007). Influence of food soiling matrix on cleaning and disinfection efficiency on surface attached Listeria monocytogenes. Food Control 18, 1165-1171. doi: 10.1016/j.foodcont.2006.06.014

Heiman, K. E., Garalde, V. B., Gronostaj, M., Jackson, K. A., Beam, S., Joseph, L., et al. (2016). Multistate outbreak of listeriosis caused by imported cheese and evidence of cross-contamination of other cheeses. USA, 2012. Epidemiol. Infect. 144, 2698-2708. doi: 10.1017/S095026881500117X

Khadre, M. A., Yousef, A. E., and Kim, J. G. (2001). Microbiological aspects of ozone applications in food: a review. J. Food Sci. 66, 1242-1252. doi: 10.1016/j. jviromet.2008.06.004

Kostaki, M., Chorianopoulos, N., Braxou, E., Nychas, G. J., and Giaouris, E. (2012). Differential biofilm formation and chemical disinfection resistance of sessile cells of Listeria monocytogenes strains under monospecies and dual-species (with Salmonella enterica) conditions. Appl. Environ. Microbiol. 78, 2586-2595. doi: 10.1128/AEM.07099-11

Krysinski, E. P., Brown, L. J., and Marchisello, T. J. (1992). Effect of cleaners and sanitizers on Listeria monocytogenes attached to product contact surfaces. J. Food Prot. 55, 246-251. doi: 10.4315/0362-028X55.4.246

Mahmoud, B. S., Vaidya, N. A., Corvalan, C. M., and Linton, R. H. (2008). Inactivation kinetics of inoculated Escherichia coli O157:H7, Listeria monocytogenes and Salmonella Poona on whole cantaloupe by chlorine dioxide gas. Food Microbiol. 25, 857-865. doi: 10.1016/j.fm.2008. 05.009 
McCollum, J. T., Cronquist, A. B., Silk, B. J., Jackson, K. A., O’Connor, K. A., Cosgrove, S., et al. (2013). Multistate outbreak of listeriosis associated with cantaloupe. N. Engl. J. Med. 369, 944-953. doi: 10.1056/NEJMoa1215837

Minei, C. C., Gomes, B. C., Ratti, R. P., D'Angelis, C. E., and De Martinis, E. C. (2008). Influence of peroxyacetic acid and nisin and coculture with Enterococcus faecium on Listeria monocytogenes biofilm formation. J. Food Prot. 71, 634-638. doi: 10.4315/0362-028X-71.3.634

Moore, G., Griffith, C., and Peters, A. (2000). Bactericidal properties of ozone and its potential application as a terminal disinfectant. J. Food Prot. 63, 1100-1106. doi: 10.4315/0362-028X-63.8.1100

Norwood, D. E., and Gilmour, A. (2000). The growth and resistance to sodium hypochlorite of Listeria monocytogenes in a steady-state multispecies biofilm. J. Appl. Microbiol. 88, 512-520. doi: 10.1046/j.1365-2672.2000.00990.x

Papaioannou, E., Giaouris, E. D., Berillis, P., and Boziaris, I. S. (2018). Dynamics of biofilm formation by Listeria monocytogenes on stainless steel under monospecies and mixed-culture simulated fish processing conditions and chemical disinfection challenges. Int. J. Food Microbiol. 267, 9-19. doi: 10.1016/j. ijfoodmicro.2017.12.020

Parish, M. E., Beuchat, L. R., Suslow, T. V., Harris, L. J., Garrett, E. H., Farber, J. N., et al. (2003). Methods to reduce/eliminate pathogens from fresh and fresh-cut produce. Comp. Rev. Food Sci. Food Saf. 2, 161-173. doi: 10.1016/j.ijfoodmicro. 2015.07.015

Pitts, B., Hamilton, M. A., Zelver, N., and Stewart, P. S. (2003). A microtiter-plate screening method for biofilm disinfection and removal. J. Microbiol. Methods 54, 269-276. doi: 10.1016/S0167-7012(03)00034-4

Poimenidou, S. V., Chrysadakou, M., Tzakoniati, A., Bikouli, V. C., Nychas, G. J., and Skandamis, P. N. (2016). Variability of Listeria monocytogenes strains in biofilm formation on stainless steel and polystyrene materials and resistance to peracetic acid and quaternary ammonium compounds. Int. J. Food Microbiol. 237, 164-171. doi: 10.1016/j.ijfoodmicro.2016.08.029

Rice, R. G., and Graham, D. M. (2001). US FDA regulatory approval of ozone as an antimicrobial agent-what is allowed and what needs to be understood. Ozone News 29, 22-31.

Robbins, J. B., Fisher, C. W., Moltz, A. G., and Martin, S. E. (2005). Elimination of Listeria monocytogenes biofilms by ozone, chlorine, and hydrogen peroxide. J. Food Prot. 68, 494-498. doi: 10.4315/0362-028X-68.3.494
Saa Ibusquiza, P., Herrera, J. J., and Cabo, M. L. (2011). Resistance to benzalkonium chloride, peracetic acid and nisin during formation of mature biofilms by Listeria monocytogenes. Food Microbiol. 28, 418-425. doi: 10.1016/j.fm.2010. 09.014

Scallan, E., Hoekstra, R. M., Angulo, F. J., Tauxe, R. V., Widdowson, M. A., Roy, S. L., et al. (2011). Foodborne illness acquired in the United Statesmajor pathogens. Emerg. Infect. Dis. 17, 7-15. doi: 10.3201/eid1701. P11101

Sheng, L., Hanrahan, I., Sun, X., Taylor, M. H., Mendoza, M., and Zhu, M. J. (2018). Survival of Listeria innocua on Fuji apples under commercial cold storage and ozone gaseous. Food Microbiol. 76, 21-28. doi: 10.1016/j.fm.2018.04.006

Silk, B. J., Date, K. A., Jackson, K. A., Pouillot, R., Holt, K. G., Graves, L. M., et al. (2012). Invasive listeriosis in the foodborne diseases active surveillance network (FoodNet), 2004-2009: further targeted prevention needed for higherrisk groups. Clin. Infect. Dis. 54(Suppl. 5), S396-S404. doi: 10.1093/cid/ cis 268

Stopforth, J. D., Samelis, J., Sofos, J. N., Kendall, P. A., and Smith, G. C. (2002). Biofilm formation by acid-adapted and nonadapted Listeria monocytogenes in fresh beef decontamination washings and its subsequent inactivation with sanitizers. J. Food Prot. 65, 1717-1727. doi: 10.4315/0362-028X-65.11.1717

Vaid, R., Linton, R. H., and Morgan, M. T. (2010). Comparison of inactivation of Listeria monocytogenes within a biofilm matrix using chlorine dioxide gas, aqueous chlorine dioxide and sodium hypochlorite treatments. Food Microbiol. 27, 979-984. doi: 10.1016/j.fm.2010.05.024

Conflict of Interest Statement: The authors declare that the research was conducted in the absence of any commercial or financial relationships that could be construed as a potential conflict of interest.

Copyright $\odot 2018$ Korany, Hua, Green, Hanrahan, El-Shinawy, El-kholy, Hassan and Zhu. This is an open-access article distributed under the terms of the Creative Commons Attribution License (CC BY). The use, distribution or reproduction in other forums is permitted, provided the original author(s) and the copyright owner(s) are credited and that the original publication in this journal is cited, in accordance with accepted academic practice. No use, distribution or reproduction is permitted which does not comply with these terms. 